\title{
SPATIO-TEMPORAL SAMPLING AND RECONSTRUCTION OF DIFFUSION FIELDS INDUCED BY POINT SOURCES
}

\author{
John Murray-Bruce and Pier Luigi Dragotti \\ Imperial College London \\ Electrical and Electronic Engineering Department \\ e-mail:\{john.murray-bruce07, p.dragotti\}eimperial.ac.uk
}

\begin{abstract}
In this paper we consider a diffusion field induced by multiple point sources and address the problem of reconstructing the field from its spatio-temporal samples obtained using a sensor network. We begin by formulating the problem as a multi-source estimation problem - so estimating source locations, activation times and intensities given samples of the induced field. Next a two-step algorithm is proposed for the single (localized and instantaneous) source field. First, the source location and intensity are estimated by applying the "reciprocity gap" principle; we show that this step can also reveal locations of multiple non-instantaneous sources. In the second step, we use an iterative method, based on CauchySchwarz inequality, to find the activation time given the estimated location and intensity. Finally we extend this algorithm to the multi-source field and present simulation results to validate our findings.
\end{abstract}

Index Terms - Spatio-temporal sampling, sensor network, diffusion process, reciprocity gap, Prony's method

\section{INTRODUCTION}

Numerous biological and physical phenomena are modeled by the diffusion equation: temperature variation in fluids, disease epidemic dynamics, population dispersion and biochemical substance release are typical examples of such phenomena. Whilst the use of sensor networks to obtain spatiotemporal samples of these physical phenomena is becoming increasingly commonplace, the space-time dimensions of these diffusion processes are generally inhomogeneous. Thus regular multidimensional sampling theory [1] can in general no longer be applied. A robust and efficient solution to this sampling and reconstruction problem will strongly impact several real-life applications such as, pollution detection [2], environmental monitoring [3] and energy efficiency monitoring in large data center clusters $[4,5]$.

In this paper, we will consider the problem of sampling and reconstructing fields governed by the diffusion equation.

This work is supported by the European Research Council (ERC) starting investigator award Nr. 277800 (RecoSamp).
In other words we will attempt to infer the entire field distribution given a limited number of observations obtained using a sensor network. Such a problem is widely known to be illposed [6], but can be regularized by imposing a model on the sources of the field. This approach transforms the problem into a parametric source estimation problem. Indeed works in the area have focused mainly on source localization. For example, Lu and Vetterli proposed two methods for source localization, namely spatial super-resolution [7] and an adaptive sampling scheme [8]. A localization method based on $L_{1}$ constrained optimization is introduced in [9]. Ranieri et al proposed a compressed sensing approach [10], whilst Auffray et al proposed a method based on the reciprocity gap [11]. Ranieri and Vetterli [12] have also considered uniform spatial sampling and reconstruction using classical interpolation techniques. While Rostami et al [13] introduced diffusive compressive sensing (DCS) to solve the problem.

In this paper, we propose a source estimation-based method - that is we fully estimate the sources inducing the field and rely on the premise that fully specifying these sources allows for complete field reconstruction. We assume multiple spatially localized sources and take advantage of the reciprocity gap principle [11] to estimate the initial source intensities and locations, whilst the activation times are retrieved by performing a simple linear search. Given that we have access only to sparse field measurements, we adapt the reciprocity gap method to operate properly within this new context. In particular, contrary to common practice [11, 14], we utilize sensor measurements both along and inside an arbitrary domain boundary to perform source localization using measurements taken over a short time interval. This allows estimation of intensities, locations and activation times of active sources with high accuracy, even in the presence of noise. Furthermore, we also propose the use of a new test function that improves the stability of the localization step.

The rest of this paper is organized as follows. Section 2 formally discusses the sampling and reconstruction problem in the source estimation setting. Section 3 presents the reciprocity gap method for source intensity and location retrieval, and we propose a linear search method to estimate the ac- 
tivation time; we conclude the section by combining these solutions to give a single source estimation algorithm. In Section 4 we generalize the single source algorithm to the multiple-source case. We validate our findings through numerical simulations in Section 5 and conclude the paper in Section 6.

\section{PROBLEM FORMULATION}

In what follows we will formulate the diffusion field sampling and reconstruction problem. Firstly let us consider an unknown point source distribution $f$ within a region $\Omega$, that induces a diffusion field $u$. Such a phenomenon is governed by the diffusion equation;

$$
\frac{\partial}{\partial t} u(\mathbf{x}, t)=\mu \nabla^{2} u(\mathbf{x}, t)+f(\mathbf{x}, t)
$$

where $\mu$ is the diffusivity of the medium through which the field propagates, $\mathbf{x} \in \mathbb{R}^{d}$ denotes the spatial domain, whilst $t$ is the temporal domain. Moreover, from the theory of Green's functions a solution to this PDE is [15]:

$$
u(\mathbf{x}, t)=(g * f)(\mathbf{x}, t),
$$

where $g(\mathbf{x}, t)=\frac{1}{(4 \pi \mu t)^{d / 2}} e^{-\frac{\|\mathbf{x}\|^{2}}{4 \mu t}} H(t)$ is the Green's function of the diffusion field, and $H(t)$ is the unit step function. The implication of Equation (2) is such that finding $f$ given discrete measurements of $u$ means the entire field can be perfectly reconstructed. We consider the case where the sources are localized in space and concentrated in time leading to the following source parameterization:

$$
f(\mathbf{x}, t)=\sum_{m=1}^{M} c_{m} \delta\left(\mathbf{x}-\boldsymbol{\xi}_{m}, t-t_{m}\right),
$$

where $c_{m}, \boldsymbol{\xi}_{m}, t_{m}$ is the concentration, location and activation time respectively of the $m$-th source in a field induced by $M$ sources.

Hence the sampling and reconstruction problem is equivalent to finding the source parameters $\left\{c_{m}, \boldsymbol{\xi}_{m}, t_{m}: m=\right.$ $1, \ldots, M\}$ given spatial and temporal samples of the diffusion field $u$. In our analysis we consider the 2-D problem $(d=2)$ and assume that we have access to field measurements $\varphi_{n}\left(t_{l}\right)=u\left(\mathbf{x}_{n}, t_{l}\right)$, obtained at instants $t_{l}$ for $l=0, \ldots, L$ and from sensors at spatial locations $\mathbf{x}_{n}$ for $n=1, \ldots, N$ situated along an arbitrary domain boundary $\partial \Omega$ and its enclosed region $\Omega$. We note that the domain boundary $\partial \Omega$ may be arbitrarily chosen provided all active sources are contained within its domain $\Omega$ (see Figure 1). For simplicity however, our simulations will consider a circular boundary with sensors evenly distributed along the boundary and uniformly distributed within the region enclosed. We also briefly address the transient source localization problem, i.e. finding the locations $\left\{\boldsymbol{\xi}_{m}: m=1, \ldots, M\right\}$ for sources with distribution:

$$
f(\mathbf{x}, t)=\sum_{m=1}^{M} c_{m} e^{\alpha_{m}\left(t-t_{m}\right)} \delta\left(\mathbf{x}-\boldsymbol{\xi}_{m}\right) H\left(t-t_{m}\right),
$$

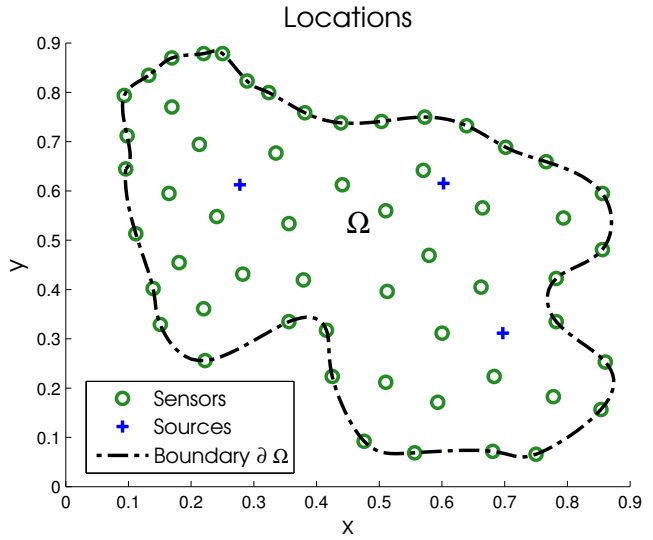

Fig. 1. Sensor placement along and within the boundary.

where $\alpha_{m}<0$ is the decay coefficient.

\section{DIFFUSION SOURCE ESTIMATION}

In this section we first use reciprocity gap functional (RGF) theory to derive a Vandermonde system which can be solved for the intensities and locations of multiple point sources. Next we address the single source activation time estimation problem given estimates of its intensity and location. Finally we present the single source estimation algorithm.

\subsection{Multiple Source Localization and Intensity Estima- tion}

Reciprocity gap functionals are derived by comparing a field with its adjoint $\psi[16,17]$. In our setting $\psi$ must satisfy the time-reversed diffusion equation:

$$
\frac{\partial \psi}{\partial t}+\mu \nabla^{2} \psi=0 \quad \text { in } \Omega .
$$

Moreover, for the domain $\Omega$ with a sufficiently smooth boundary $\partial \Omega$ Green's second identity may be used to relate the field at the boundary to that inside the domain as follows:

$\int_{\Omega} \frac{\partial}{\partial t}(\psi u) \mathrm{d} V-\mu \oint_{\partial \Omega}(\psi \nabla u-u \nabla \psi) \cdot \hat{\mathbf{n}}_{\partial \Omega} \mathrm{d} S=\int_{\Omega} \psi f \mathrm{~d} V$

where $\hat{\mathbf{n}}_{\partial \Omega}$ is the outward pointing unit normal vector to $\partial \Omega$. Henceforth we shall denote the "reciprocity gap," the left hand side of Equation (6) as $R(\psi, t)$ for conciseness. Hence the reciprocity gap in time integrated form, on some interval $t \in[0, T]$ is such that $\int_{0}^{T} R(\psi, t) \mathrm{d} t=$ $\int_{\Omega} \psi u(\mathbf{x}, T) \mathrm{d} V-\mu \oint_{\partial \Omega}(\psi \nabla U-U \nabla \psi) \cdot \hat{\mathbf{n}}_{\partial \Omega} \mathrm{d} S$, where $U(\mathbf{x})=\int_{0}^{T} u(\mathbf{x}, t) \mathrm{d} t$. Therefore

$$
\int_{0}^{T} R(\psi, t) \mathrm{d} t=\int_{0}^{T} \int_{\Omega} \psi f \mathrm{~d} V \mathrm{~d} t
$$


Combining Equations (3) and (7) yields $\int_{0}^{T} R(\psi, t) \mathrm{d} t=$ $\int_{0}^{T} \int_{\Omega} \psi \sum_{m=0}^{M} c_{m} \delta\left(\mathbf{x}-\boldsymbol{\xi}_{m}, t-t_{m}\right) \mathrm{d} V \mathrm{~d} t$. We thus set $\psi \rightarrow \Psi_{k}(\mathbf{x})=e^{-k\left(x_{1}+j x_{2}\right)}$, where $k=0,1, \ldots, K$, and this results in the Vandermonde system:

$$
\mathcal{R}(k)=\sum_{m=1}^{M} c_{m} e^{-k\left(\xi_{1, m}+j \xi_{2, m}\right)}, k=0,1, \ldots, K
$$

where $\mathcal{R}(k)=\int_{0}^{T} R\left(\Psi_{k}, t\right) \mathrm{d} t$. This system can then be solved using Prony's method [18]. Indeed this choice of test function, a decaying complex exponential rather than a complex polynomial, improves the numerical stability of the source localization step.

Disposed with the spatial samples (in $\Omega$ and along $\partial \Omega$ ) as well as temporal samples (over $[0, T]$ ) of the diffusion field, it is possible to obtain an estimate of the quantity $\int_{0}^{T} R\left(\Psi_{k}, t\right) \mathrm{d} t$ by properly combining the sensor measurements $\left\{\varphi_{n}\left(t_{l}\right): n=1, \ldots, N, l=0, \ldots, L\right\}$. Specifically in the 2D case, the boundary integral is estimated using standard quadrature methods [19], similarly the surface integral may be estimated using the methods described by Georg in $[20,21]$. Taking the surface integral into account, in contrast to Auffray et al who assume it decays to zero [11], allows us to operate on measurements taken over short time windows. In so doing, we are able to retrieve the source locations with better accuracy. Moreover, most approaches assume a physical boundary, accompanied by boundary conditions. In our case the domain boundary is artificial and defined by the sensor locations.

Remark 1. We obtain a similar result to Equation (8) when the source is transient. Indeed a similar analysis to that above using Equation (4) yields:

$$
\mathcal{R}(k)=\sum_{m=1}^{M} c_{m}^{\prime} e^{-k\left(\xi_{1, m}+j \xi_{2, m}\right)},
$$

where $c_{m}^{\prime}=\frac{c_{m}}{\alpha_{m}}\left(e^{\alpha_{m}\left(T-t_{m}\right)}-1\right)$.

\subsection{Single Source Activation Time Retrieval}

Assuming a single instantaneous source field $(M=1)$ where the concentration $(c)$ and location $(\boldsymbol{\xi})$ of the source have already been estimated using Equation (8), we propose a simple iterative "linear search" solution to find the activation time parameter $(\tau)$ of the source as follows: first consider the sequence of field measurements $\left\{\varphi_{n}\left(t_{l}\right): l=0, \ldots, L\right\}$ made by the $n$-th sensor (- situated at $\mathbf{x}_{n}$ ). Moreover, let us make an initial "guess" $\tau_{0}$ at the activation time of the single source field, hence for this $\tau_{0}$ the reconstructed field at $\mathbf{x}_{n}$ is $\hat{u}\left(\mathbf{x}_{n}, t-\tau_{0}\right)=\frac{c}{4 \pi \mu\left(t-\tau_{0}\right)} e^{-\frac{\left\|\mathbf{x}_{n}-\boldsymbol{\xi}\right\|^{2}}{4 \mu\left(t-\tau_{0}\right)}} H\left(t-\tau_{0}\right)$ and its sampled version $\left\{\hat{\varphi}_{n}\left(t_{l}, \tau_{0}\right)=\hat{u}\left(\mathbf{x}_{n}, t_{l}-\tau_{0}\right): l=0, \ldots, L\right\}$. Compare the measured and reconstructed sequences (in vector form) for several values of $\tau_{0}$ and choose the $\tau_{0}$ that generates a reconstructed sequence most similar to the measured one. We achieve this by maximizing the normalized inner product between both sequences - a modification of the Cauchy-Schwarz inequality for vectors.

\subsection{Single Source Estimation Algorithm}

In Algorithm 1 we formally present the two-step algorithm for estimating the parameters of a single diffusion source from samples of its induced field. In the first step the RGF method is used to estimate the source's intensity and location; in the second step a selection of the nearest sensors are each independently used to estimate the activation time and their average taken to be an improved estimate of the activation time.

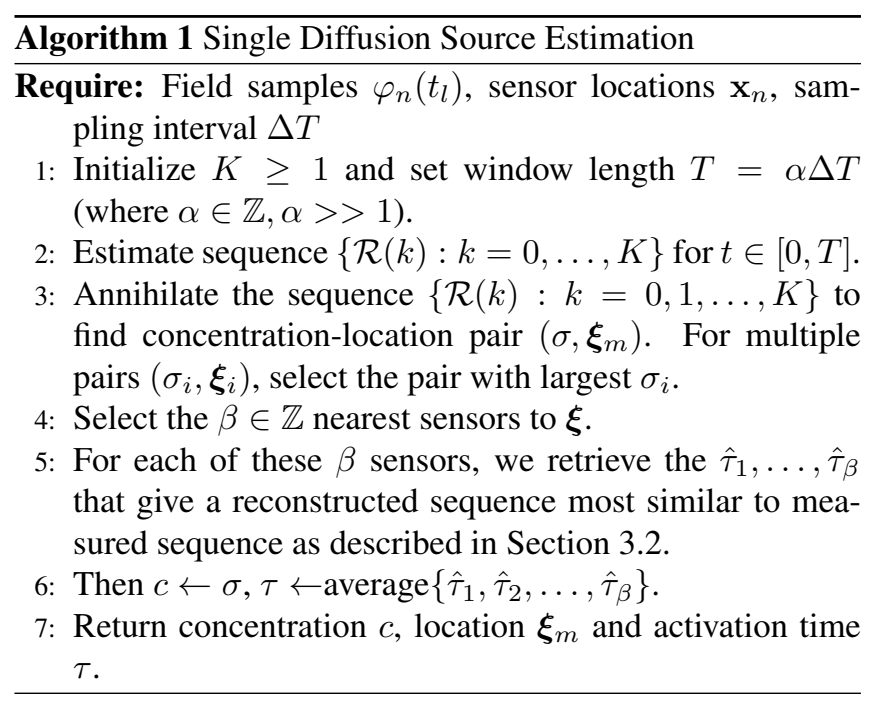

\section{MULTIPLE SOURCE ESTIMATION ALGORITHM}

Algorithm 1 provides a systematic way of estimating a single point and instantaneous source from "boundary" and "interior" samples of its field. In fact it is easily generalized to the multiple source case provided the sources are well separated in time - specifically, the sampling interval is assumed to be small enough to resolve the activation of two consecutive sources. The modification is as follows:

1. Using Equation (8) search for two sources (choose $K \geq 3$ ) over some time window of length $T=\alpha \Delta T$ that is much smaller than the duration $\left(T_{\text {end }}\right)$ over which the entire field is sampled (so $T<<T_{\text {end }}$ ).

2. If one only source is found (either $\sigma_{2} \approx 0$ or $\xi_{2} \notin$ $\Omega$ ) then run steps 4 onwards of Algorithm 1, otherwise decrement (say $\alpha=\alpha-1$ ) the time window (and repeat steps 1 and 2 above) until only one source is found.

3. Now reconstruct the field due to the current source at all sensor locations, for the duration of the samples taken. 

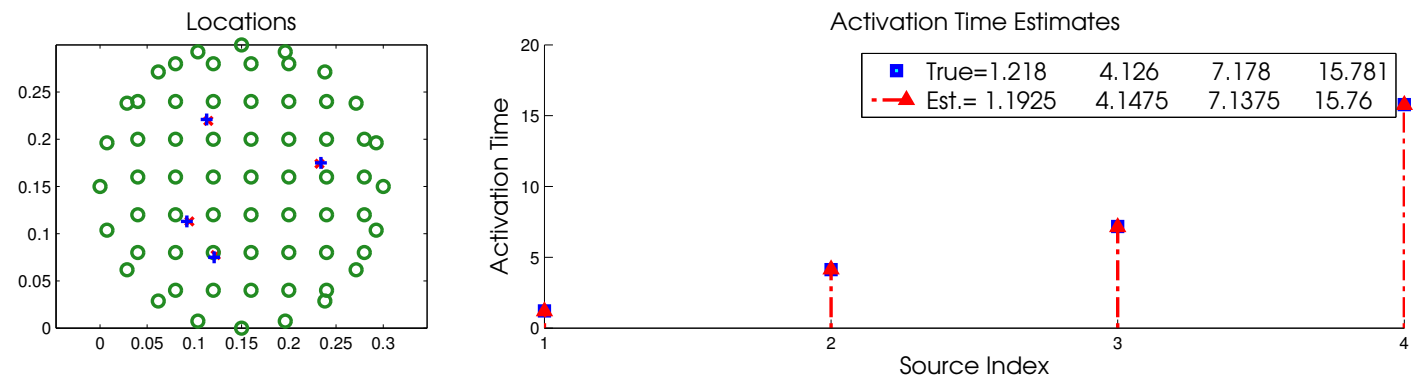

(a) Ideal (noiseless) measurement samples.
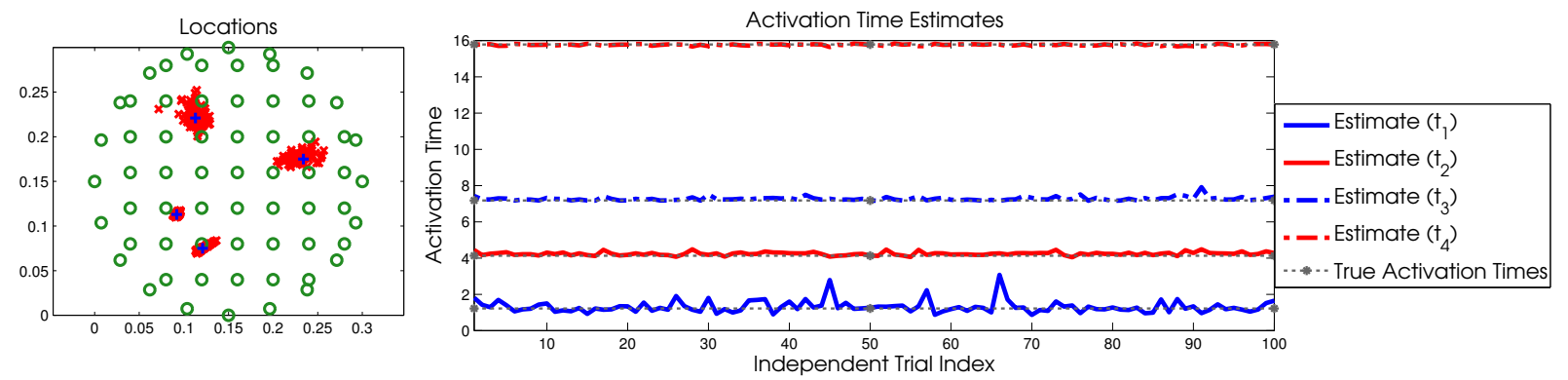

(b) 100 independent trials using noisy sensor measurement samples (SNR=15dB).

Fig. 2. Source Estimation from circular (radius $=0.15 \mathrm{~m}$ ) boundary and uniform ( $\mathrm{spacing}=0.04 \mathrm{~m}$ ) interior measurements. The scatter-plots show sensor locations (green ' $O$ '), true source locations (blue ' + ') and estimated locations (red ' $X$ ').

4. Remove its contribution from the original measurements to get the "adjusted" measurement and repeat with a larger time window. We stop when the adjusted measurement is below a predetermined threshold.

This algorithm is evaluated in Section 5 using synthetic field measurements.

\section{SIMULATION RESULTS}

In this section we provide simulation results showing the performance of our multi-source estimation algorithm. We simulate the 2-D field governed by the diffusion equation, in particular we consider the setting where the field is induced by four sources activated at different times. Samples of the field are then collected, at $1 \mathrm{~Hz}$ for 25 seconds, by sensors arranged along a circular boundary $(\partial \Omega)$ and uniformly inside the bounded region $(\Omega)$. The simulation parameters are summarized below:

- $M=4$. Intensities $c_{m}=1$ for $m=1, \ldots, 4$. Locations $\boldsymbol{\xi}_{1}=(0.113,0.221), \boldsymbol{\xi}_{2}=(0.234,0.175), \boldsymbol{\xi}_{3}=$ $(0.121,0.075), \boldsymbol{\xi}_{4}=(0.092,0.113)$. For activation times see legend in Figure 2a.

- Field sampled over $T_{\text {end }}=25$ seconds at sampling frequency $1 / \Delta T=1 \mathrm{~Hz}$.

- $K=3$, i.e. $k=0,1, \ldots, 3$ for the test function family $\Psi_{k}(\mathbf{x})=e^{-k\left(x_{1}+j x_{2}\right)}$
- 43 Interior Sensors and 20 Boundary sensors.

Figure 2 demonstrates the ability of the proposed algorithm to successfully estimate the location and activation times of the active sources. In addition, we retrieve the following estimates, $\hat{c}_{1}=0.9758, \hat{c}_{2}=1.0207, \hat{c}_{3}=0.9833$ and $\hat{c}_{4}=0.9509$, for the source intensities given ideal measurements. For the noisy measurements $(\mathrm{SNR}=15 \mathrm{~dB})$, the concentration estimates vary marginally around these estimates for each source (with occasional spikes in the range $0.8-1.2)$. We have also observed that the estimation accuracy is dependent on the number of interior sensors to a higher degree, more interior sensors increases the estimation accuracy and also reduces the spread of the estimates in the noisy setting. The boundary sensors however have little effect on the estimation accuracy.

\section{CONCLUSION}

In this paper an algorithm for solving the diffusion source estimation problem in 2-D using boundary and interior measurements of the induced field is presented. In particular we solve the estimation problem when the sources are spatiallylocalized and instantaneous. Simulations demonstrate that the estimation algorithm is noise robust even in the multiple source setting, thanks to the averaging effects from the time integrated field, as well as the averaging of the multiple activation time estimates from the nearest sensors to the source. 


\section{REFERENCES}

[1] D. P. Petersen and D. Middleton, "Sampling and reconstruction of wavenumber-limited functions in ndimensional euclidean spaces," Information and Control, vol. 5, no. 4, pp. 279 - 323, 1962.

[2] A. El Badia and T. Ha-Duong, "On an inverse source problem for the heat equation. application to a pollution detection problem," Journal of Inverse and Ill Posed Problems, vol. 10, no. 6, pp. 585-600, 2002.

[3] G. Barrenetxea, F. Ingelrest, G. Schaefer, M. Vetterli, O. Couach, and M. Parlange, "Sensorscope: Out-ofthe-box environmental monitoring.," in Information Processing in Sensor Networks(IPSN), St. Louis, USA, April 2008.

[4] B. Ricardo and R. Ram, "Power and energy management for server systems," IEEE Computer, vol. 37, pp. 2004, 2004.

[5] K. Chen, D. M. Ausl, C. E. Bash, and R. D. Patel, "Local temperature control in data center cooling," Hewlett Packard Laboratories, Tech. Rep. HPL-2006-42, 2006.

[6] P. C. Hansen, Rank-Deficient and Discrete Ill-Posed Problems: Numerical Aspects of Linear Inversion, vol. 4, SIAM, 1987.

[7] Y. M. Lu and M. Vetterli, "Spatial super-resolution of a diffusion field by temporal oversampling in sensor networks," in Proc. IEEE International Conference on Acoustics, Speech and Signal Processing (ICASSP). IEEE, 2009, pp. 2249-2252.

[8] Y. M. Lu and M. Vetterli, "Distributed spatio-temporal sampling of diffusion fields from sparse instantaneous sources," in Computational Advances in Multi-Sensor Adaptive Processing (CAMSAP), 2009 3rd IEEE International Workshop on. IEEE, 2009, pp. 205-208.

[9] Y. Li, S. Osher, and R. Tsai, "Heat source identification based on L1 constrained minimization," UCLA CAM report 11-04, 2011.

[10] J. Ranieri, A. Chebira, Y. M. Lu, and M. Vetterli, "Sampling and reconstructing diffusion fields with localized sources," in Proc. IEEE International Conference on Acoustics, Speech and Signal Processing (ICASSP). IEEE, 2011, pp. 4016-4019.

[11] N. Auffray, M. Bonnet, and S. Pagano, "Identification of transient heat sources using the reciprocity gap," Inverse Problems in Science and Engineering, vol. 21, no. 4, pp. 721-738, 2013.
[12] J. Ranieri and M. Vetterli, "Sampling and reconstructing diffusion fields in presence of aliasing," in Proc. IEEE International Conference on Acoustics, Speech and Signal Processing (ICASSP). IEEE, 2013, pp. 5474-5478.

[13] M. Rostami, N.-M. Cheung, and T. Q. S. Quek, "Compressed sensing of diffusion fields under heat equation constraint," in Proc. IEEE International Conference on Acoustics, Speech and Signal Processing (ICASSP). IEEE, 2013, pp. 4271-4274.

[14] Z. Dogan, I. Jovanovic, T. Blu, and D. Van De Ville, "3D reconstruction of wave-propagated point sources from boundary measurements using joint sparsity and finite rate of innovation," in 9th Proc. IEEE International Symposium on Biomedical Imaging (ISBI)., may 2012, pp. $1575-1578$.

[15] V. I. Yukalov, Statistical Green's Functions, Queen's papers in pure and applied mathematics. Queen's University, 1998.

[16] P. Steinhorst and A.-M. Sändig, "Reciprocity principle for the detection of planar cracks in anisotropic elastic material," Inverse Problems, vol. 28, no. 8, pp. 085010, 2012.

[17] Z. Dogan, V. Tsiminaki, I. Jovanovic, T. Blu, and D. Van De Ville, "Localization of point sources for systems governed by the wave equation," in Proc. of SPIE Vol, 2011, vol. 8138, pp. 81380P-1.

[18] P.-L. Dragotti, M. Vetterli, and T. Blu, "Sampling moments and reconstructing signals of finite rate of innovation: Shannon meets strang-fix," IEEE Transactions on Signal Processing, vol. 55, no. 5, pp. 1741-1757, 2007.

[19] G. Dahlquist and Å. Björck, Numerical Methods in Scientific Computing: Vol. 1, Number v. 1 in SIAM ebooks. Society for Industrial and Applied Mathematics (SIAM, 3600 Market Street, Floor 6, Philadelphia, PA 19104), 2008.

[20] K. Georg, "Approximation of integrals for boundary element methods," SIAM journal on scientific and statistical computing, vol. 12, no. 2, pp. 443-453, 1991.

[21] K. Georg and J. Tausch, "Some error estimates for the numerical approximation of surface integrals," mathematics of computation, vol. 62, no. 206, pp. 755-763, 1994. 